\title{
Treatment of neovascular age-related macular degeneration: Current therapies
}

\author{
Albert J Augustin \\ Stefan Scholl \\ Janna Kirchhof \\ Department of Ophthalmology, \\ Klinikum Karlsruhe, Germany
}

Correspondence: Albert J Augustin Department of Ophthalmology, Klinikum Karlsruhe, Moltkestrasse 90, 76133

Karlsruhe, Germany

Tel +497219742001

Fax +4972I 9742009

Email albertjaugustin@googlemail.com

\begin{abstract}
Choroidal neovascularization (CNV) secondary to age-related macular degeneration (AMD) is now the leading cause of blindness and severe vision loss among people over the age of 40 in the Western world. Its prevalence is certain to increase substantially as the population ages. Treatments currently available for the disease include laser photocoagulation, verteporfin photodynamic therapy, and intravitreal injections of corticosteroids and anti-angiogenic agents. Many studies have reported the benefits of each of these treatments, although none is without its risks. No intervention actually cures AMD, nor the neovascularization associated with it. However, its symptoms are treated with varying degrees of success. Some treatments stabilize or arrest the progress of the disease. Others have been shown to reverse some of the damage that has already been done. These treatments can even lead to visual improvement. This paper will review the major classes of drugs and therapies designed to treat this condition.
\end{abstract}

Keywords: wet AMD, neovascularization, PDT, steroids, anti-angiogenesis

\section{Introduction}

Age-related macular degeneration (AMD) was first described in the medical literature well over a century ago ${ }^{1,2}$ and yet it was not until the 1970s that an intervention was proposed. It took another 30 years for treatments to be developed that would actually arrest the progress of the disease, and in some cases, restore a degree of lost visual acuity (VA). This contrasts with other ocular diseases such as glaucoma, which was described in detail by Albrecht von Graefe more than 150 years ago. He introduced the iridectomy as a means of lowering intraocular pressure, thus providing the first effective treatment for closed angle glaucoma. It was not until 1980 that macular degeneration was reported to be a significant cause of blindness in the United States. ${ }^{3}$ Since then, many studies have expanded upon the risks associated with new blood vessel growth in the aging retina, and have shown just how serious and widespread a public health issue AMD represents.

A 2004 analysis $^{4}$ reported that among Americans over the age of 40, AMD and/or geographic atrophy were present in at least one eye in $1.47 \%$ of the population, and that 1.75 million individuals have AMD. Among women over the age of $85,15 \%$ have AMD. Approximately 7 million Americans have drusen measuring $>125$ microns. The presence of large drusen is a known risk factor for the disease, and suggests that these people could face a substantial risk of going blind. Given the aging population, it is forecast that there may be a $50 \%$ increase in the incidence of AMD before the year 2020. In another study, ${ }^{5}$ AMD was reported to account for $54 \%$ of all current cases of blindness among the Caucasian population in the United States. The study predicted that as a result of the rising prevalence of AMD, the number of blind people in the US could increase by as much as $70 \%$ by 2020 . Meanwhile, a multi-country European study ${ }^{6}$ has reported that $3.3 \%$ of the population over age 65 has grade 4 
AMD (ARM International Classification System ${ }^{7}$ ), and 2.3\% have choroidal neovascularization (CNV).

\section{Laser photocoagulation}

The earliest treatment for AMD was pan-retinal photocoagulation (PRP). This was originally used for the treatment of diabetic retinopathies. ${ }^{8}$ Key studies in the $1980 \mathrm{~s}^{9-11}$ reported that patients with $\mathrm{AMD}$, known at that time as senile macular degeneration, who were at risk of developing $\mathrm{CNV}$ were less likely to experience severe vision loss if they were treated with laser thermotherapy rather than having no treatment at all. In 1976, the Macular Photocoagulation Study (MPS) ${ }^{10}$ demonstrated a five-year event-free rate of $36 \%$ (an event was defined as the loss of six or more lines on the Early Treatment Diabetic Retinopathy Study [ETDRS] chart from baseline) for untreated patients, compared with a $54 \%$ event-free rate for patients who underwent argon laser photocoagulation. Despite the promise that laser photocoagulation offered for some patients, the benefits were inconsistent, the risks were substantial, and recurrences were frequent. In 1990, the MPS group also showed ${ }^{12}$ that when patients were treated with krypton red laser photocoagulation, one-third would have persistent neovascularization within six weeks of the initial treatment, and $47 \%$ would have recurrent neovascularization within five years. Both persistent and recurrent disease were associated with severe vision loss.

In the Moorsfield study ${ }^{11}$ in Britain, in 1982, there was a numerical superiority in terms of the number of patients whose visual acuity improved when they were treated with argon laser. However, the difference between treated and untreated groups was only significant at the $p=0.05$ level among those who had the worst visual acuity (VA) at baseline. The differences were not significant among patients who had better baseline VA (6/24 or 6/36).

There are other important limitations to laser photocoagulation. It destroys the overlying retina, and therefore can cause significant and permanent loss of some vision. It cannot be employed where there is subfoveal CNV leakage, and must be used not less than 200 microns outside the fovea. It is applicable only for lesions that can be clearly defined in the early phase of fluorescein angiography, which rules out its use for occult neovascularization. In total, laser photocoagulation may only be appropriate for approximately $15 \%$ of potential patients with $\mathrm{CNV} .{ }^{13}$

\section{Intravitreal corticosteroids}

Numerous investigators over the years have reported that a significant factor in the pathogenesis of AMD is inflammation.
It has therefore been suggested that corticosteroids could play a role in treating or preventing the inflammatory process. There is good rationale for the use of steroids in treating CNV. Besides reducing inflammation, steroids block the up-regulation of vascular endothelial growth factor (VEGF), which is produced and released by all inflammatory cells. ${ }^{14}$ Corticosteroids are thought to reduce vascular leakage because they close up the gaps that form between endothelial cells in the capillary walls. They also limit fibrosis, which reduces scarring in the retina.

The corticosteroid triamcinolone acetonide, administered intravitreally (IVTA) was proposed as a treatment because prior studies had shown that it could inhibit fibrovascular proliferations in rats with laser-induced $\mathrm{CNV} .{ }^{15}$ Other studies reported the same effect in primates, with safety and tolerability studies showing that it was well-tolerated in both rabbits and primates. ${ }^{16-18}$

In human trials, IVTA was shown to stabilize the progress of AMD as seen on fundus photography and fluorescein angiography, and/or to improve visual acuity. ${ }^{19-21}$ Those studies used $4 \mathrm{mg}$ doses of triamcinolone; one study ${ }^{22}$ however, investigated the use of repeat injections of $25 \mathrm{mg}$. No unexpected adverse events were reported as a result of the higher dose, although the improvements in visual acuity were not significantly better than those seen when the lower dose (4 mg) was used. In any case, not all triamcinolone studies produced encouraging results. Gillies and colleagues ${ }^{23}$ followed patients who received a single injection of $4 \mathrm{mg}$ IVTA vs patients who received a placebo for a minimum period of one year. The study revealed a significant difference in the size of the neovascular membrane favoring patients who received the steroid at the end of three months, but this difference disappeared by the end of the one-year follow-up. Moreover, $35 \%$ of both treated and untreated patients experienced a loss of $>30$ ETDRS letters. Besides showing little real benefit for triamcinolone compared with placebo, the study reported that $41 \%$ of patients receiving IVTA had significant increases in intraocular pressure compared with $4 \%$ of patients in the control group. Similar increases in intraocular pressure (IOP) were also seen in subsequent trials, ${ }^{24,25}$ and have come to represent a substantial risk for patients who have, or are at risk of developing open angle glaucoma.

A second steroid, dexamethasone, has a more or less similar anti-angiogenic effect to triamcinolone. However, it is shorter-acting, and is administered in solution form, rather than as a suspension. Therefore it is cleared from the vitreous faster than triamcinolone. Dexamethasone does not reduce the trabecular drain as severely as triamcinolone, 
so it is not associated with the spikes in IOP that are seen with triamcinolone use.

\section{Verteporfin photodynamic therapy}

Photodynamic therapy with verteporfin (V-PDT) entails a two-part process with the photosensitizer (verteporfin) injected intravenously first. This is followed by controlled exposure to blue laser light, at $689 \mathrm{nM}, 600 \mathrm{~mW} / \mathrm{cm}^{2}$, $50 \mathrm{~J} / \mathrm{cm}^{2}$, for 83 seconds. Neovascularization is eradicated when the verteporfin, which accumulates in the choroidal vessels, is activated by the laser light, and generates reactive oxygen species. These attach to localized endothelial cells, causing platelet binding and aggregation. This blocks further blood flow through the vessels, and in fairly quick order, atrophy of the neovascularization follows. Blood vessels that have been eradicated in this way do not grow back, although other vessels will still be formed within the subretinal space due to continued expression of VEGF.

V-PDT gained rapid acceptance following its release in North America and Europe in 1999. Initially it was approved for the treatment of subfoveal, predominantly classic AMD lesions of all sizes. Indications have expanded to include occult with no classic lesions, certain minimally classic lesions, lesions of $\leq 4$ MPS disk areas (DA), and CNV secondary to pathological myopia.

The first trial to report the benefits of V-PDT was the Treatment of Age-related Macular Degeneration with Photodynamic Therapy (TAP) study. ${ }^{26}$ The protocol used in this study continues to inform the use of this agent.

The TAP study comprised two separate randomized double-masked placebo-controlled clinical trials involving a total of 609 patients who had predominantly classic AMD with CNV. At 12 months follow-up, 61\% of patients in the treatment arm lost $<15$ letters vs $46 \%$ in the placebo arm. At 24 months follow-up, $53 \%$ and $38 \%$ of the two groups respectively lost $<15$ letters. More than twice as many patients in the V-PDT group had a complete absence of CNV leakage compared with the placebo group. These subjects have now been followed out to more than five years, during which they received an average of 7.6 treatments. The most recently published paper ${ }^{27}$ reported minimal changes in visual acuity between months 24 and 60 . Approximately $21 \%$ of patients lost 1 to 3 lines at two years, and the same percentage was followed out to year 5 . Of the patients with predominantly classic lesions, $41 \%$ had a loss of 6 lines (30 letters) or less at month 24 , compared with $55 \%$ at month 60 . The authors caution that there were many factors, including a substantial rate of discontinuation, which could have caused these numbers to appear artificially low. Also, since there was no control group in years 3 to 5 , it is not known whether subjects who were treated did any better than untreated subjects would have done between months 24 and 60 .

A study of 339 patients with occult with no classic neovascularization ${ }^{28}$ reported that after two years follow-up, 54\% and $30 \%$ of V-PDT treated patients lost $\geq 15$ letters and $\geq 30$ letters of VA respectively, compared with $67 \%$ and $47 \%$ of patients who received a sham treatment. A sub-group analysis found that patients were more likely to demonstrate stable vision, or even a visual gain at 24 months follow-up when they had worse vision ( $\leq 65$ letters or $20 / 200$ ) or lesions of $\leq 4$ disk areas diameter at baseline.

A further subset analysis revealed an interesting and highly useful fact about V-PDT treatments and patient response rates. ${ }^{29}$ While the above trials looked at all lesion characteristics, multiple linear regression modeling revealed a significant correlation between treatment outcomes and lesion size for minimally classic and occult with no classic lesions, but not for predominantly classic lesions. Smaller lesions were associated with less loss of visual acuity, regardless of lesion composition. Thus, lesion size at baseline ( $\leq 4.0 \mathrm{DA}$, whether minimally classic, or occult with no classic) was determined to be more predictive of outcome than either lesion composition or baseline visual acuity.

The first randomly-controlled double-masked study investigating the safety and efficacy of V-PDT in patients with minimally classic $\mathrm{CNV}^{30}$ produced mixed results. This study involved 117 patients randomized to either the $\mathrm{V}$-PDT at a reduced fluence rate $\left(\mathrm{RF}\right.$, ie, $300 \mathrm{~mW} / \mathrm{cm}^{2}$ for 83 seconds at $25 \mathrm{~J} / \mathrm{cm}^{2}$ ), V-PDT at the standard fluence (SF, ie, $600 \mathrm{~mW} / \mathrm{cm}^{2}$ for 83 seconds, at $50 \mathrm{~J} / \mathrm{cm}^{2}$ ) or placebo. Patients had lesions of $\leq 6$ DA. While the difference in the mean change in VA from baseline favored the RF arm over the SF arm at 12 and 24 months, these differences were minor (usually less than one line) and not particularly robust compared with placebo. However, significantly more patients $(28 \%)$ in the placebo arm progressed to predominantly classic lesions during the follow-up period compared with patients in the V-PDT groups (5\% and 3\% for RF and $\mathrm{SF}$ groups respectively). Because of these inconclusive results, the Royal College of Ophthalmologists in Britain has recommended that V-PDT not be used for minimally classic lesions. In the United States, it has been recommended in cases where there are minimally classic lesions with signs of recent disease progression.

Although numerous studies continue to be conducted with V-PDT, these above-noted trials paved the way for much 
greater use of this intervention, and still represent the most important evidence-based research to justify the use of this procedure for treating AMD with CNV.

As a result of these studies, V-PDT has been shown to be safe and effective for treating a range of lesion types, including predominantly classic lesions, $\mathrm{CNV}$ secondary to pathological myopia, and occult with no classic subfoveal lesions. A combined analysis ${ }^{29}$ of the two main V-PDT studies referenced here ${ }^{26,27}$ showed that V-PDT can be used to treat lesions of $\leq 4$ DA. And finally, subfoveal minimally classic lesions which meet additional criteria may, in some cases, be managed successfully with V-PDT.

\section{Anti-angiogenic factors}

As the human eye ages, choroidal vascular atrophy and a number of other factors lead to oxidative stress and hypoxia, which result in local inflammation in the sub-retinal layers. This inflammation causes an up-regulation of VEGF, a protein which among other pro-angiogenic factors stimulates the formation of new blood vessels. ${ }^{31}$ VEGF also upregulates the production and release of matrix metallo-proteinases (MMP) 2 and 9. This degrades the extracellular matrix and leads to endothelial leakage, as well as vascular endothelial cell migration and proliferation. CNV then penetrates Bruch's membrane and the retinal pigment epithelium (RPE), leading to vision loss.

At this time, three anti-angiogenic drugs (VEGF-inhibitors) are available to treat neovascularization. The first to enter the marketplace was pegaptanib sodium, a 28-base anti-VEGF aptamer, which antagonizes the specific amino acid isoform 165 when administered intravitreally, and blocks new blood vessel growth.

The pivotal study with pegaptanib sodium was the multicenter VEGF Inhibition Study in Ocular Neovascularization (VISION trial) $^{32}$ which enrolled 1190 patients. They were treated with either $0.3 \mathrm{mg}, 1.0 \mathrm{mg}$, or $3.0 \mathrm{mg}$ of pegaptanib every six weeks $(n=892)$, or with a sham injection. The study included all lesion subtypes: predominantly classic, minimally classic, and occult with no classic CNV. At 54 weeks follow-up, patients treated with pegaptanib showed significant clinical benefits compared with patients in the placebo group. Overall, $70 \%$ of patients treated with pegaptanib sodium experienced a vision loss of $<15$ ETDRS letters, compared with $55 \%$ of patients who received a sham injection $(\mathrm{p}<0.001)$. No difference in outcomes was detected among patients who received either $1.0 \mathrm{mg}$ or $3.0 \mathrm{mg}$ of the drug compared with $0.3 \mathrm{mg}$. The treatment effect was consistent regardless of baseline visual acuity, lesion sub-type or whether the lesions were $\geq 4$ or $\leq 4$ DA.
The introduction of pegaptanib was followed closely by the emergence of two other more effective VEGF inhibitors: ranibizumab and bevacizumab. The use of bevacizumab as an ocular anti-angiogeneic is controversial, especially for the company that developed the drug (Genentech Inc., South San Francisco CA, USA). It was originally intended for use in the treatment of colorectal cancer, namely to inhibit metastatic angiogenesis. Ranibizumab, also developed by Genentech, is significantly more costly, but any greater efficacy remains to be proven. Bevacizumab has not been approved for ocular use in any country by any licensing authorities; nonetheless, it is widely used off-label to treat AMD and other conditions of the eye. Bevacizumab is a full-length, humanized monoclonal antibody (149 Kd molecular weight) which, unlike pegaptanib, is directed against all biologically active isoforms of VEGF-A. Both drugs are derived from the same mouse antibody against VEGF; ranibizumab is thought to have a greater binding affinity for VEGF owing to its smaller molecular weight $(48 \mathrm{Kd})$.

Published studies involving patients treated with $1.25 \mathrm{mg}$ bevacizumab ${ }^{33,34}$ injections spaced one month apart, have demonstrated significant improvements in retinal thickness in as little as one week after the first injection, and significant improvements in VA, which are defined by a halving of the visual angle have also been observed at three months. Such improvements appear to be sustained over several months.

The typical dose of bevacizumab is $1.25 \mathrm{mg}$. A small study $^{35}$ that included intravitreal injections of $2.5 \mathrm{mg}$ enrolled 17 patients who either were not candidates for, or had failed V-PDT therapy. Significant improvements in VA $(p<0.001)$ from baseline at four and eight weeks could be observed. No appreciable improvement in VA was noted beyond that. In the absence of a comparator group, however, it remains impossible to say whether there was any benefit to treating these patients with the higher dose.

No significant adverse events or other safety issues have been identified in any of the studies with bevacizumab. This is an important consideration given that when the drug is used in the oncology setting, the side effects include delayed wound healing, increased hypertension, and gastric bleeding and perforations.

A third monoclonal antibody against VEGF, and the second to be approved for the treatment of AMD-associated $\mathrm{CNV}$, is ranibizumab. Like bevacizumab, ranibizumab binds all isoforms of VEGF-A. Both are derived from the same mouse antibody against VEGF, but while bevacizumab is the full-length antibody, ranibizumab is only a Fab fragment of it. 
The main published study ${ }^{36}$ with ranibizumab (Minimally Classic/Occult Trial Age-Related Macular Degeneration, or MARINA) was a two-year, phase III trial designed to evaluate monthly injections in 716 patients with minimally classic or occult with no classic lesions. All of these patients had recurrent disease. They were randomized 1:1:1 to receive either sham injections $(n=238)$, or injections of ranibizumab at $0.3 \mathrm{mg}(\mathrm{n}=238)$ or $0.5 \mathrm{mg}(\mathrm{n}=240)$. The 12-month primary endpoint analysis revealed that at least $94 \%$ of patients in both arms receiving ranibizumab lost fewer than 15 letters, compared with $62 \%$ of patients in the sham treatment arm. These results were also carried out to 24 months. A gain in VA of at least 15 letters was observed in $25 \%$ of patients in the $0.3 \mathrm{mg}$ ranibizumab arm, and $34 \%$ of patients in the $0.5 \mathrm{mg}$ ranibizumab arm. The gains in visual acuity occurred regardless of lesion type or size, or baseline VA, and were evident within seven days of the first injection.

The next study ${ }^{37}$ (Anti-VEGF Antibody for the Treatment of Predominantly Classic Choroidal Neovascularization in Age-Related Macular Degeneration; ANCHOR) compared the same two dosage strengths of ranibizumab against V-PDT in 423 patients who were followed out to 12 months. At that point, patients in the ranibizumab $0.5 \mathrm{mg}$ arm gained, on average, 11.3 letters, while patients in the V-PDT arm lost, on average, 9.5 letters, for an overall difference in treatment effect of 20.8 ETDRS letters. The outcomes were more or less similar at month 24 . In addition, approximately $95 \%$ of patients in both ranibizumab arms lost fewer than 15 letters, while $35 \%$ and $40 \%$ of patients in the $0.3 \mathrm{mg}$ and $0.5 \mathrm{mg}$ arms respectively gained more than 15 letters compared with patients who were treated with V-PDT. Interestingly, a sub-group analysis ${ }^{38}$ reported that as lesion size increased, the benefit of ranibizumab $0.5 \mathrm{mg}$ became less significant when compared with V-PDT.

In general, ranibizumab was well-tolerated. Adverse events that did occur were more frequent in patients receiving the higher $0.5 \mathrm{mg}$ dose. The most frequent ocular adverse events included local injection site and ocular inflammation, as well as spikes in intraocular pressure, which could be significant but appeared to be transient. Nonocular hemorrhage was seen more often in patients receiving ranibizumab compared with the other two groups. Arteriothrombolic events, which include nonfatal myocardial infarction and/or stroke, and death from other vascular causes, were seen in 3.8\% of patients receiving $0.3 \mathrm{mg}$ of ranibizumab and $4.6 \%$ of those receiving the $0.5 \mathrm{mg}$ dose. These rates did not differ from patients who received the sham injection. Rosenfeld and colleagues $^{39}$ investigated several doses of ranibizumab and recommended $0.5 \mathrm{mg}$ as the optimal dose.

An issue that has generated no small amount of controversy, in both the medical literature and in the daily press, has to do with the cost of ranibizumab. Its price is listed ${ }^{40}$ at US\$1950 wholesale per dose. For a drug that requires monthly injections, this may represent a considerable burden for many patients. Bevacizumab, which has the same mechanism of action, and only a slightly different molecular structure, is said to cost US\$17-\$50 per injection, but it is used off-label. Patients are therefore faced with the dilemma of being treated with a very expensive drug which has been approved for use in the United States and many other countries, but may require indefinite monthly injections, or a similar drug that is much cheaper, but is not approved for ocular use. The National Eye Institute is now conducting, at its own expense, a head-to-head trial to determine whether there is a difference in efficacy between these two drugs.

To help address the issue of dealing with the cost of monthly treatments, trials have been carried out to investigate different dosing regimens with ranibizumab. ${ }^{41,42}$ At first glance, the rationale for flexible dosing, or "as needed dosing" appears to make sense. Because VEGF inhibitors have a short half-life, their effects wear off fairly quickly. Regular injections of ranibizumab could therefore be needed indefinitely. In the two main ranibizumab trials cited here ${ }^{36,37}$ most of the benefit occurred within the first three months of treatment. The improvements in VA between months 3 and 12 were modest at 1.8 and 1.5 letters for the two studies, respectively. Therefore, it was reasoned that patients might be able to achieve significant gains in VA within the first three months, and that additional treatments could be given on an "only as needed" basis.

In the Prospective Optical Coherence Tomography Imaging of Patients with Neovascular AMD Treated with intra-Ocular ranibizumab (PrONTO) trial ${ }^{41} 40$ patients received ranibizumab $0.5 \mathrm{mg}$ at baseline, and then again at months 1 and 2 . They did not receive additional treatments unless certain pre-specified criteria were met. These criteria included a loss of $\geq 5$ ETDRS letters, and/or an increase in macular thickness of at least 100 microns, continued subretinal fluid detected by optical coherence tomography (OCT) after one month, new hemorrhage, and new neovascularization. An analysis of 37 patients produced encouraging data in terms of reduced retinal thickening, and improved VA, but for a number of reasons, these results cannot be generalized to clinical practice. First, this was a single center trial, involving only 40 patients. Also, patients in this study had all types 
of lesions, unlike subjects in either of the other studies. ${ }^{36,37}$ Moreover, this was an open-label, unmasked study, in which patients had received prior therapies.

In a second study, ${ }^{42}$ (A Phase IIIb, Multicenter, Randomized, Double-Masked, Sham Injection-Controlled Study of the Efficacy and Safety of Ranibizumab in Subjects with Subfoveal Choroidal Neovasularization with or without Classic CNV Secondary to Age-Related Macular Degeneration; PIER) patients received injections of ranibizumab $0.3 \mathrm{mg}$ or $0.5 \mathrm{mg}$, or a sham, at baseline, and then again at months 1 and 2. Further injections were given at three-month intervals (ie, months 5 , 8,11 ), for a total of six injections over 11 months. Initially, patients receiving the higher dose of $0.5 \mathrm{mg}$ ranibizumab had a 4.3 letter gain in VA over the first three months (ie, while monthly injections were being given), but this gain quickly deteriorated once quarterly dosing was commenced. At the end of 12 months, these patients had a mean decrease in VA of 0.2 letters from baseline. Along with the loss in VA, the patients also experienced renewed vascular leakage as seen on fluorescein angiography, and increases in mean retinal thickness as seen on OCT. These data suggest that neither quarterly, nor "as needed" dosing of ranibizumab is useful in anything but the short term, and that monthly injections are likely needed to ensure any kind of viable treatment effect. Additionally, OCT findings should be considered for the adjustment of the dosing regimen.

In general, few serious adverse events have been reported following ocular treatment with anti-VEGF drugs, with the exception of case reports of RPE tears following anti-VEGF administration. There is a wide variance in the incidence of these tears, ranging from less than one percent ${ }^{43,44}$ to $17 \%{ }^{45}$, and because of the size of the studies, these numbers are relatively low. Nonetheless, these are important events and can result in significant, permanent loss of vision.

Most cases of RPE tear occur in the presence of pigment epithelial detachment (PED) lesions, and it appears that fibrovascular lesions are more susceptible to RPE tears than serous lesions. This suggests that either these patients have very fragile RPE structure, that they had a more severe form of CNV, or perhaps that they are somehow predisposed to weakened RPE, or already had pre-existing PED. Chiang and colleagues ${ }^{45}$ have reported that the risk of developing an RPE tear correlates directly with the diameter and height of the PED size, as well as with the presence of subretinal fluid as seen on OCT.

\section{Combination therapy}

Each of the interventions discussed in this paper has its own particular strengths and limitations, but no single treatment offers a perfect solution or a cure for AMD-associated CNV. Augustin and colleagues have had some notable success by combining bevacizumab, V-PDT, and the steroid dexamethasone into a "triple therapy regimen" that attacks CNV through three different mechanisms. V-PDT eradicates existing neovascularization. Bevacizumab, the anti-angiogenic, prevents the growth of new blood vessels in the eye. And finally, besides being an anti-inflammatory, the corticosteroid dexamethasone has anti-angiogenic properties and antifibrotic and anti-permeability characteristics, which help preserve the integrity of the blood-retinal barrier.

In Augustin's interventional case series, ${ }^{46} 104$ patients with all types of choroidal neovascularization were treated with V-PDT at a reduced light dose of 70 seconds, intravitreal dexamethasone $(800 \mu \mathrm{g})$, and bevacizumab $(1.25 \mathrm{mg})$. The mean VA improved from 20/126 at baseline to 20/85 at 40 weeks follow-up. This corresponds to an improvement of 1.8 ETDRS lines or 8.9 letters. There was also a significant decrease in retinal thickness, from 463.5 microns at baseline, to 281 microns at 40 weeks follow-up. This indicates that both functional and anatomical improvements were seen with this triple therapy regimen. These results have now been carried out to 70 weeks follow-up.

\section{Customized therapies}

Each of the treatments reported so far concentrates mainly on one aspect of AMD-associated CNV (for example laser photocoagulation on the newly grown vessels) and therefore has its strengths and its weaknesses. Augustin's triple therapy regimen offers another attractive alternative for treatment, because it relies on three different mechanisms of action: eradicating existing CNV (vaso-occlusive), preventing new $\mathrm{CNV}$ (anti-angiogenic), and countering the inflammatory process.

With that in mind, we herein see the great advantage of a therapy tailored to address not only the specific characteristics of a patient's disease (ie, minimally classic or predominantly classic lesions), but the patient's personal circumstances as well. Customized therapies should offer a more efficacious intervention, a treatment that poses less of a burden for the patient personally or financially, and one that is less of a burden for the caregivers and for the healthcare systems in general. There are many citations in the literature that deal with "individualized therapy", but these are predominantly in diseases such as cancer, diabetes, psychiatric/emotional disorders, hypertension and HIV/AIDS. The only application where one has spoken of customized therapy in the ophthalmology context has been certain studies that have 
investigated combination of ocular hypotensive drugs to lower IOP in glaucoma patients.

Ophthalmologists now have a good understanding of the pathophysiology and biochemistry behind CNV, but we must build upon this knowledge in order to develop such customized therapies. We already employ treatments with various doses of laser light and VEGF inhibitors, but at this time, we do not have an algorithm to inform us of which patient may respond best to a particular therapy, or whether a patient may respond better to more of one agent, and less of another. Even something as simple as delivering photodynamic therapy and ranibizumab on the same day, rather than on two separate occasions, is being investigated. ${ }^{47}$ According to preliminary data, same-day administration may result in less endophthalmitis and uveitis compared with treatments delivered days apart. Reductions in lesion dimensions and central retinal thickness were also observed. ${ }^{48}$

Finding the elusive "customized treatment" is not the same as finding a new drug, although a long list of new drugs with novel mechanisms of action are being developed. These new interventions will act more "upstream" and will address the issue of oxidative stress, drusen formation, inflammation, and other steps in the cascade that lead to new vessel growth and vision loss. Such pharmacologic approaches may focus on genetic factors such as the $\mathrm{Y} 402 \mathrm{H}$ polymorphism which lead to an inappropriate inflammatory response at the level of the complement system. Several complement cascade inhibitors are now under development. Other long-range pharmacologic interventions could include drugs to boost, or augment naturally occurring factors that antagonize angiogenesis. These factors are known to include pigment-derived epithelial factor and endostatin.

\section{Conclusion}

During the past four decades there have been some highly encouraging and much needed improvements in the management of CNV secondary to AMD. Early treatments like laser photocoagulation prevented the rapid spread of $\mathrm{CNV}$, but often led to some degree of permanent vision loss. V-PDT arrested the progress of the disease and even improved visual acuity for a small percentage of patients. Anti-angiogenic agents (VEGF inhibitors) now offer improvements in visual acuity for at least one-third of patients. Apart from VEGF, many other anti-angiogenic factors exist and may be rewarding targets of future studies. Those studies should not only aim to identify additional important factors being involved in the pathogenesis of the disease entity but also investigate new treatment approaches.
Each of the developments discussed here has been built upon the strengths of its predecessors, continually improving the effective treatment of CNV secondary to AMD. These improvements will become even more significant as the population ages and the prevalence of vision loss due to AMD becomes more widespread.

\section{Acknowledgments}

The authors wish to thank Cameron Johnston (London, Canada) for his assistance in preparing this paper for publication. The authors report no conflicts of interest in this work.

\section{References}

1. Haab O. Erkrankungen der macula Lutea. Zentralbl Augenheilkd. 1885;9:384-391.

2. Nettleship E. Central areolar choroidal atrophy. Trans Ophthalmol Soc UK. 1884;4:165-166.

3. Leibowitz HM, Krueger DA, Maunder RA, et al. The Framingham Eye Study monograph; an ophthalmological study of cataract, glaucoma, diabetic retinopathy, macular degeneration and visual acuity in a general population of 2631 adults 1973-75. Surv Ophthalmol. 1980;24(Suppl):335-610.

4. Friedman DS, O'Colmain BJ, Muñoz B, et al; Eye Diseases Prevalence Research Group. Prevalence of age-related macular degeneration in the United States. Arch Ophthalmol. 2004;122:564-572.

5. Congdon N, O'Colmain B, Klaver CC; the Eye Diseases Prevalence Research Group. Causes and prevalence of visual impairment among adults in the United States. Arch Ophthalmol. 2004;122:477-485.

6. Augood CA, Vingerling JR, de Jong PTVM, et al. Prevalence of agerelated maculopathy in older Europeans. The European Eye Study (EurEye). Arch Ophthalmol. 2006;124:529-535.

7. Bird AC, Bressler NM, Bressler SB, et al. An international classification and grading system for age-related maculopathy and age-related macular degeneration. The International ARM Epidemiological Study Group. Surv Ophthalmol. 1995;39:367-374.

8. Preliminary report on effects of photocoagulation therapy. The Diabetic Retinopathy Study Research Group. Am J Ophthalmol. 1976;81: 383-396.

9. Coscas G, Soubrane G. Photocoagulation des neo vaisseaux sous-retiniens dans la degenerescence maculaire senile par laser a argon; resultats de l'etude randomisee de 60 cas. Bull Soc Ophtalmol Fr. 1982;94:149-154.

10. Argon laser photocoagulation for senile macular degeneration. Results of a randomized clinical trial. Arch Ophthalmol. 1982;100:912-918.

11. Treatment of senile disciform macular degeneration: a single-blind randomised trial by argon laser photocoagulation. The Moorfields Macular Study Group. Br J Ophthalmol. 1982;66:745-753.

12. Persistent and recurrent neovascularization after krypton laser photocoagulation for neovascular lesions of age-related macular degeneration. Macular Photocoagulation Study Group. Arch Ophthamol. 1990;108:825-831.

13. Freund KB, Yannuzzi LA, Sorenson JA. Age-related macular degeneration and choroidal neovascularization. Am J Ophthalmol. 1993;115:786-791.

14. Möhle R, Green D, Moore MA, Constitutive production and thrombininduced release of vascular endothelial growth factor by human megakaryocytes and platelets. PNAS. 1997;94:663-668.

15. Ciulla TA, Criswell MH, Danis RP. Intravitreal triamcinolone acetonide inhibits choroidal neovascularization in a laser-treated rat model. Arch Ophthalmol. 2001;119:399-404.

16. Chandler DB, Rozakis G, de Juan E Jr. The effect of triamcinolone acetonide on a refined experimental model of proliferative vitreoretinopathy. Am J Ophthalmol. 1985;99:686-690. 
17. Hida T, Chandler D, Arena JE. Experimental and clinical observations of the intraocular toxicity of commercial corticosteroid preparations. Am J Ophthalmol. 1986;101:190-195.

18. Ishibashi T, Miki K, Sorgente N, Patterson R. Effects of intravitreal administration of steroids on experimental subretinal neovascularization in the subhuman primate. Arch Ophthalmol. 1985;103:708-711.

19. Challa JK, Gillies MC, Penfold PL, Exudative macular degeneration and intravitreal triamcinolone: 18 month follow up. Aust N ZJ Ophthalmol. 1998;26:277-281.

20. Penfold PL, Gyory JF, Hunyor AB Exudative macular degeneration and intravitreal triamcinolone. A pilot study. Aust N Z J Ophthalmol. 1995;23:293-298

21. Danis RP, Ciulla TA, Pratt LM. Intravitreal triamcinolone aceotonide in exudative age-related macular degeneration. Retina. 2000;20:244-250.

22. Jonas JB, Akkoyun I, Budde WM. Intravitreal reinjection of triamcinolone for exudative age-related macular degeneration. Arch Ophthalmol. 2004;122:218-222.

23. Gillies MC, Simpson JM, Luo W. A randomized clinical trial of a single dose of intravitreal triamcinolone acetonide for neovascular age-related macular degeneration: one-year results. Arch Ophthalmol. 2003;121:667-673.

24. Jonas JB, Degenring RF, Kreissig I. Intraocular pressure elevation after intravitreal triamcinolone acetonide injection. Ophthalmology. 2005;112:593-598.

25. Rhee DJ, Peck RE, Belmont J. Intraocular pressure alterations following intravitreal triamcinolone acetonide. Br J Ophthalmol. 2006;90: 999-1003.

26. Photodynamic therapy of subfoveal choroidal neovascularization in age-related macular degeneration with verteporfin: one-year results of 2 randomized clinical trials - TAP report. Treatment of age-related macular degeneration with photodynamic therapy (TAP) Study Group. Arch Ophthalmol. 1999;117:1329-1345.

27. Kaiser PK; Treatment of Age-related Macular Degeneration with Photodynamic Therapy (TAP) Study Group. Verteporfin therapy of subfoveal choroidal neovascularization in age-related macular degeneration: 5-year results of two randomized clinical trials with an openlabel extension: TAP report no. 8. Graefes Arch Clin Exp Ophthalmol. 2006;244:1132-1142.

28. Verteporfin In Photodynamic Therapy Study Group. Verteporfin therapy of subfoveal choroidal neovascularization in age-related macular degeneration: two-year results of a randomized clinical trial including lesions with occult with no classic choroidal neovascularization-verteporfin in photodynamic therapy report 2. Am J Ophthalmol. 2001;131:541-560.

29. Blinder KJ, Bradley S, Bressler NM, et al; Treatment of Age-related Macular Degeneration with Photodynamic Therapy study group; Verteporfin in Photodynamic Therapy study group. Effect of lesion size, visual acuity, and lesion composition on visual acuity change with and without verteporfin therapy for choroidal neovascularization secondary to age-related macular degeneration: TAP and VIP report no. 1. Am J Ophthalmol. 2003;136:407-418.

30. Azab M, Boyer DS, Bressler NM, et al; Visudyne in Minimally Classic Choroidal Neovascularization Study Group. Verteporfin therapy of subfoveal minimally classic choroidal neovascularization in age-related macular degeneration: 2-year results of a randomized clinical trial. Arch Ophthalmol. 2005;123:448-457.
31. Kannan R, Zhang N, Sreekumar PG, et al. Stimulation of apical and basolateral VEGF-A and VEGF-C secretion by oxidative stress in polarized retinal pigment epithelial cells. Mol Vis. 2006;12:1649-1659.

32. Gragoudas ES, Adamis AP, Cunningham ET Jr. Pegaptanib for neovascular age-related macular degeneration. $N$ Engl J Med. 2004;351: 2805-2816.

33. Rich RM, Rosenfeld PJ, Puliafito CA. Short-term safety and efficacy of intravitreal bevacizumab (Avastin) for neovascular age-related macular degeneration. Retina. 2006;26:495-511.

34. Spaide RF, Laud K, Fine HF. Intravitreal bevacizumab treatment of choroidal neovascularization secondary to age-related macular degeneration. Retina. 2006;26:383-390.

35. Bashshur ZF, Bazarbachi A, Schakal A. Intravitreal bevacizumab for the management of choroidal neovascularization in age-related macular degeneration. Am J Ophthalmol. 2006;142:1-9.

36. Rosenfeld PJ, Brown DM, Heier JS. Ranibizumab for neovascular agerelated macular degeneration. $N$ Engl J Med. 2006;355:1419-1431.

37. Brown DM, Kaiser PK, Michels M. Ranibizumab versus verteporfin for neovascular age-related macular degeneration. $N$ Engl J Med. 2006;355:1432-1444.

38. Kaiser PK, Brown DM, Zhang K. Ranibizumab for predominantly classic neovascular age-related macular degeneration: Subgroup analysis of first-year ANCHOR results. Am J Ophthalmol. 2007;144:850-857.

39. Rosenfeld PJ, Heier JS, Hantsbarger G, Shams N. Tolerability and efficacy of multiple escalating doses of ranibizumab (Lucentis) for neovascular age-related macular degeneration. Ophthalmology. 2006;113:623-632.

40. Steinbrook R. The price of sight-ranibizumab, bevacizumab, and the treatment of macular degeneration. $N$ Engl J Med. 2006;355: 1409-1412.

41. Fung AE, Lalwani GA, Rosenfeld PJ. An optical coherence tomography-guided, variable dosing regimen with intravitreal ranibizumab (Lucentis) for neovascular age-related macular degeneration. Am J Ophthalmol. 2007;143:566-583.

42. Regillo CD, Brown DM, Abraham P. Randomized, double-masked, shamcontrolled trial of ranibizumab for neovascular age-related macular degeneration: PIER Study Year 1. Am J Ophthalmol. 2008;145:239-248.

43. Heier JS; the ANCHOR Study Group. Ranibizumab: Two-year ANCHOR Study results. Oral presentation. Retina Subspecialty Day, Annual Meeting of American Academy of Ophthalmology, New Orleans, USA; 2007.

44. Paolo L. Lessons Learned from the FOCUS Trial. Oral presentation. Retina Subspecialty Day, Annual Meeting of American Academy of Ophthalmology, New Orleans, USA; 2007.

45. Chiang A, Chang LK, Yu F. Predictors of anti-VEGF-associated retinal pigment epithelial tear using FA and OCT analysis. Retina. 2008 Jul 14. [Epub ahead of print].

46. Augustin AJ, Puls S, Offermann I. Triple therapy for choroidal neovascularization due to age-related macular degeneration: verteporfin PDT, bevacizumab, and dexamethasone. Retina. 2007;27:113-140.

47. Callanan DG. Unpublished study. Oral presentation. Retina Subspecialty Day, Annual Meeting of American Academy of Ophthalmology, New Orleans, USA; 2007.

48. Schmidt-Erfurth U, Wolf S. Paper submitted to Archives of Ophthalmology. Oral presentation. Retina Subspecialty Day, Annual Meeting of American Academy of Ophthalmology, New Orleans, USA; 2007. 